\title{
Towards Semantic Interoperability for Electronic Health Records
}

\section{Domain Knowledge Governance for openEHR Archetypes}

\author{
S. Garde', P. Knaup ${ }^{2}$, E. J. S. Hovenga' ${ }^{1}$ S. Heard', 3 \\ 'Central Queensland University, Health Informatics Research Group, Faculty of Business \\ and Informatics, Melbourne, Victoria, and Rockhampton, Queensland, Australia \\ 2University of Heidelberg, Department of Medical Informatics, Heidelberg, Germany \\ ${ }^{3} 0$ cean Informatics, Australia
}

\section{Introduction}

The "Integrated Care Electronic Health Record" (EHR) is defined by the International Organization for Standardization (ISO) [1] as " ... a repository of information regarding the health of a subject of care in computer processable form, stored and transmitted securely, and accessible by multiple authorised users. It has a commonly agreed logical information model which is independent of EHR systems. Its primary purpose is the support of continuing, efficient and quality integrated health care and it contains information which is retrospective, concurrent and prospective".

In practical terms, this means that an EHR has the following characteristics:

- The EHR is patient-centred: one EHR relates to one subject of care, not to an episode of care at an institution.

- The EHR is longitudinal: it is a longterm record of care, possibly birth to death.

- The EHR is comprehensive: it includes a record of care events from all types of carers, providers and institutions tending to a patient, not just one specialty; in other words there are no important care events of any kind not in the EHR.

- The EHR is prospective: not only are previous events recorded, but also instructions and prospective information such as plans, goals, orders and evaluations.

A myriad of reasons ranging from physician concerns about workflow to broad environmental issues are still inhibiting the adoption of EHR systems [2]. Some argue the most important reason why clinicians are reluctant to adopt clinical IT systems is a perceived lack of added value [3]. The UK Royal College of Nursing finds in a recent study that $93 \%$ of nurses believe that training for EHRs is very important, but over $50 \%$ received no training [4]. Clinicians commonly feel that it is others who benefit from their keyboard labours - health system administrators, payors, and, hopefully, the patient [3]. But to really add value for the clinician it is still often necessary to develop best-of-breed systems (e.g. [5]). Frequently, these systems then circumvent existing systems with a strong administrative focus in one form or another. Usually, best-of-breed systems are at best awkwardly integrated, often maintained with minimum resources, and are not interoperable with other systems. Further, nowadays, health care provider organisations and individual health professionals typically are highly specialised. For optimal patient care, the various provider organisations and health professionals have to cooperate closely during patient care, often called shared care or integrated care. Shared care is defined as the continuous patient-oriented cooperation of hospitals, general practitioners (GPs), specialists and other health care professionals during patient care ([6] p. 596, [7]). The quality of patient care in shared care environments is a current research topic $([8,9])$ : Some studies suggest that shared care provides patient care as effective as a specialists-only environment, but is less expensive (e.g. [10]), whereas others (especially in the field of diabetes) find that shared care can improve the quality of care, information exchange, as well 
as adherence to evidence-based guidelines ([11-13]).

Information technology (IT) offers the potential to enable efficient communication $[14,15]$ to reduce costs [16], improve quality of care [12] and also is a means to patient empowerment [17]. In contrast, shared care imposes great challenges on the availability and processing of information including trusting shared information, the correct and clinically safe interpretation of the information, etc. If not systematically dealt with, IT can lead to more complex and variable processes imposing additional workload and sources of error on clinicians [18]. If not systematically and continuously evaluated, IT hazards and failures can reduce the quality of care [19]. Shared care requires excellent (i.e. fast and highquality) communication between the various health care providers [20]. Captured data must be of high quality (i.e. correct and complete), high reliability and high flexibility. This requires a mixture of free text entries and highly structured and standardized data items and the use of integrity constraints and plausibility checks (cp. [21, 22]). Different information systems used by the various health care providers of shared care must be able to interoperate [23], so that one system can understand the context and meaning of information provided by another system (semantic interoperability).

The openEHR archetype methodology (http://www.openEHR.org) is a possible solution to the described "Shared-CareDilemma" as it claims to empower the clinician and ensure seamless integration and semantic interoperability.

\section{Objectives}

The aim of this paper is to answer the following research questions:

- What impact do openEHR and archetypes have on health professionals?

- What impact do openEHR and archetypes have on semantic interoperability?

To do this, we will initially briefly present the openEHR and archetype approach. We will then analyse the impact of archetypes on health professionals, define various levels of interoperability and analyse how openEHR and archetypes contribute to these interoperability levels. For this, we will also explore the relationship of archetypes to clinical terminologies like SNOMED CT and analyse the openEHR approach with regard to its compliance to existing or developing standards. In this context, we will define what domain knowledge governance is, why it is needed to ensure semantic interoperability in health care, and how it can be accomplished on the basis of openEHR archetypes. We then investigate how this process can best be supported by information technologies. Finally, real world examples of its use are presented. The openEHR Blood Pressure Archetype is one of the most comprehensively investigated of all openEHR archetypes and will be used in this paper as an exemplar to present some of the key characteristics of this approach.

\section{Material and Methods}

\subsection{Nature of the Analysis}

We analysed current approaches to EHR systems, terminology and standards developments. In addition to literature reviews, we organised face-to-face and additional telephone interviews and teleconferences with members of ISO/TC 215, EHR Taskforce of European Committee for Standardization (CEN), EHR SIG of HL7 International, Standards Australia Electronic Health Record Committee, and founding members of the openEHR Foundation.

\subsection{The openEHR and Archetypes Approach}

The openEHR approach (http://www. openEHR.org) is defined in comprehensive open specifications for EHR systems originally based on the results of the European Union's GEHR-Project in the early 1990s. Following GEHR several projects extended and refined its results (e.g. the Australian GEHR projects and the EU Synapses and
SynEx projects). All these projects influenced the openEHR architecture and the pioneering of a two-level modelling approach for EHRs. The first level of this approach is the reference information model, which is pared down to the minimum to support the medico-legal requirements and record management functions. This ensures that clinicians can always send information to another provider and receive information which they can read - thus ensuring data interoperability. The second level involves the openEHR archetype methodology - a way of sharing evolving clinical information so that it can be processed by the receiving provider - thus enabling semantic interoperability. A blood pressure archetype for example represents a description of all the information a clinician might want or has to report about a blood pressure measurement. Basically, one archetype models or represents one clinical or other domain-specific concept by constraining instances of the openEHR information models to express a valid structure, valid data types, and values.

Design principles of openEHR are described in far more detail by the open specifications available from the openEHR homepage, but the key innovation of the openEHR architecture is that it separates record keeping concerns from clinical data collection using archetypes [24].

\subsection{Information Technology}

To support domain knowledge governance with information technology, we employed the Web Ontology Language (OWL [25]) and the Protégé OWL Plug-In [26] to develop and maintain an Archetype Ontology which provides the necessary meta-information on archetypes for Domain Knowledge Governance as detailed later in this paper. In addition, we used Borland JBuilder $X^{\circledR}$ as Integrated Development Environment, Java ${ }^{\mathrm{TM}}$ Servlet 2.3 Technology, and Apache Tomcat 4.1.31 as servlet container. 


\section{Results}

\subsection{The Impact of Archetypes on Health Professionals}

The proposed way of openEHR to deal with constantly changing knowledge in health care is to "put the clinicians back into the driver's seat" [3], i.e. to empower the health professionals to define and alter the accurate knowledge and information they need in the granularity they need. The openEHR archetype methodology empowers domain experts to create and change the knowledge inherent in archetypes and the openEHR Archetype Editor ${ }^{\mathrm{a}}$ renders the abstract openEHR archetype model tangible for clinicians. Archetypes can among others be presented in a HTML rendition which is automatically generated by the Archetype Editor from the archetype. Archetypes can also be used as the formal and computerprocessable specification of clinical content and can be seen as the building blocks of the health knowledge environment.

\subsubsection{Archetypes Enable the Formal Definition of Clinical Content by Domain Experts without the Need for Technical Understanding}

Archetypes are agreed models of clinical or other domain-specific concepts. From a technical point of view archetypes are formal specifications of clinical content. From a clinical point of view, archetypes serve an intuitive means to define and discuss and present clinical content.

Once defined by clinicians, archetypes can then be machine-processed and do not need interpretation by system developers, thus providing a more streamlined process from the clinicians into a clinical system. Terms within an archetype can come from any number of terminologies or, if highly specific to the archetyped concept, be specified within the archetype itself. Archetypes specify groups of data that are whole, discreet, highly related and clinically meaning-

\footnotetext{
a Available from http://www.openEHR.org or http://www.oceaninformatics.biz
}

ful. They enable information to be specified in a very powerful form and enable the clinicians to define the knowledge they need at the level of granularity required.

\subsubsection{Archetypes Conserve the Meaning of Data by Explicitly Specified and Well- structured Clinical Content}

The structure or data model of health information systems is often seen as being an important part of the intellectual property of the system vendor and kept secret. However, data stored in certain fields are displayed within particular contexts on the screen. For example, a simple two-value blood pressure of 120/80 mmHg may be displayed (and hence implicitly interpreted) as a point in time measurement of the patient's blood pressure, an average over 12 hours in 24- hour blood pressure monitoring, or a target to aim for with a range of interventions in the management of hypertension. A blood pressure archetype would formalize this interpretation and thus conserve the meaning of the data. Maximum, minimum and mean over 8 and 24 hours as typically used in 24-hour measurements can easily be formalized using an archetype-based approach. OpenEHR archetypes structure the clinical content, e.g. by providing state and protocol for observations (e.g. the patient position during a blood pressure measurement), a state machine for action archetypes (e.g. describing the pathway of a medication order from prescribed to completed) thus providing a good basis for wellstructured clinically relevant content models.

\subsubsection{Archetypes Can Safely Evolve and thus Deal with Ever-changing Health Knowledge Using a Two-level Approach}

It is claimed that the openEHR approach and archetypes empower the domain expert and deal with the ever-changing and growing health environment by using a two-level approach that separates information structures and (clinical) knowledge [3]. Strictly differentiating between these two levels is necessary because health care according to Alan Rector [27] is constantly changing in three ways:
- in breadth, because new knowledge is always being discovered or becoming relevant (e.g. SNOMED CT - the Systematized Nomenclature of Medicine Clinical Terms July 2006 edition features over 300,000 active atomic concepts with unique meanings, more than 700,000 English language descriptions or synonyms for flexibility in expressing clinical concepts),

- in depth, because finer-grained detail is always being discovered or becoming relevant,

- in complexity, because new relationships are always being discovered or becoming relevant (e.g. between a drug and adverse events). SNOMED CT for example contains more than 900,000 defining relationships.

Thomas Beale has argued that "[m]any of today's information systems are developed in such a way that the domain concepts which the system is to process are hardcoded directly into its software and database models" resulting in "systems which are expensive to modify and extend, and consequently have a limited lifespan" [28]. The adoption of archetypes facilitates knowledge domain experts (clinicians) to specify content models for clinical concepts such as blood pressure, an ECG result or a medication order, by strictly separating domain knowledge from runtime information and design time models that collectively make up an information system. This also facilitates EHR systems to accommodate for changing medical and health service delivery practices over time.

\subsubsection{Archetypes Can Simplify the Use of Clinical Terminologies}

An example how archetypes can simplify the use of terminologies is the ability to add a term 'position of the patient' to the archetyped concept of a blood pressure measurement. This requires a small term set which will include 'sitting', 'standing', 'lying'. All these terms can then easily be post-coordinated with the blood pressure measurement itself. Explicit terms like 'sitting systolic blood pressure' and 'standing systolic blood pressure' are no longer required. The 
advantage is that in the setting of a blood pressure measurement it is clear what these terms mean-compared to an all purpose list of body positions that can be found in a terminology like SNOMED-CT. Since any translation to different languages occurs within the well-specified context of one archetype only, translating an archetype is comparatively easy and the meaning of the archetype can be preserved across languages. This also helps in making multilingual and cross-cultural interoperability [29] achievable.

\subsection{Syntactic Interoperability, Semantic Interpretability, and Semantic Interoperability with openEHR Archetypes}

The Institute of Electrical and Electronics Engineers defines interoperability as the "ability of two or more components to exchange information and to use the information that has been exchanged" [30]. In the following, we differentiate in harmony with Sheth between three levels of interoperability [31]:

1) Syntactic (data) interoperability: The structure and provenance of information or knowledge is understood by a (clinical) system. This comprises all (machine-readable) aspects of data representation. Syntax can be considered as the grammar to convey semantics and structure.

2) Structural interoperability/semantic interpretability: The semantics of the information or knowledge provided is explicit and can be analysed by domain experts (clinicians). This involves (health) concept representation.

3) Semantic interoperability: Apart from comprising syntactic and structural interoperability, semantic interoperability requires that the information system understands the semantics of information request and those of information sources. Semantics is defined as the meanings of terms and expressions. Hence according to Veltman [29] semantic interoperability is "the ability of information systems to exchange information on the basis of shared, pre- established and negotiated meanings of terms and expressions."

Table 1 shows how these three levels of interoperability can be achieved by the use of openEHR and archetypes. In summary, archetypes are syntactically interoperable this is ensured by the reference model - and semantically interpretable - this is ensured by archetypes themselves. However, archetypes alone do not ensure semantically interoperable systems and archetype development must be coordinated through "Domain Knowledge Governance" to avoid incompatible archetypes for the same concept. Domain Knowledge Governance will be introduced in Section 4.3.

\subsubsection{The Relationship of openEHR and Archetypes to Clinical Terminologies like SNOMED CT}

We argue in accordance with Rector and Elkin and colleagues [27, 32] that a traditional 'comprehensive' terminology is very complex, never actually comprehensive and extremely difficult to maintain. As the openEHR archetype approach does not rely on big standardized terminologies but on "microvocabularies" [33], it offers more flexibility during standardisation and translation of clinical concepts and overcomes some of the shortcomings of terminology-focused approaches.

However, let us make the following very clear: Archetypes are not about replacing terminologies: Terms within an archetype can actually be linked (bound) to external terminologies like SNOMED-CT. Rather archetypes provide the required structure for clinical concepts and maintain two relationships to SNOMED CT:

1) Each term can be bound to a SNOMED CT concept, e.g. the diastolic blood pressure would be bound to the appropriate concept ID in SNOMED CT to specify explicitly that the archetype term and the SNOMED CT concept have the same meaning. This is in many aspects similar to the 'mapping' of OpenSDE domain models to SNOMED CT ([34]).

2) Each archetype term that is a text can be constrained by a terminology like SNOMED CT, ICD etc., e.g. the allowed values (value set) for a diagnosis text in

Table 1 Levels of interoperability and how they can be achieved using openEHR and archetypes

\begin{tabular}{|l|l|l|}
\hline $\begin{array}{l}\text { Level of } \\
\text { interoperability }\end{array}$ & $\begin{array}{l}\text { Main mechanism } \\
\text { for interoperability }\end{array}$ & Description \\
\hline $\begin{array}{l}\text { Syntactic (data) } \\
\text { interoperability }\end{array}$ & $\begin{array}{l}\text { openEHR reference } \\
\text { model (RM) }\end{array}$ & $\begin{array}{l}\text { The openEHR reference model alone ensures syntactic (data) interoperability } \\
\text { independent of any defined archetypes. The openEHR reference model does } \\
\text { not define clinical knowledge; this is defined and communicated by archetypes } \\
\text { separate from the reference model. Hence, data items are communicated } \\
\text { between systems only in terms of clearly defined, generic reference model } \\
\text { instances. As the reference model is stable, achieving syntactic interoperability } \\
\text { between systems is undemanding. }\end{array}$ \\
\hline $\begin{array}{l}\text { Structural inter- } \\
\text { operability }\end{array}$ & Archetypes & $\begin{array}{l}\text { Structural interoperability is achieved by the definition and use of archetypes. } \\
\text { As agreed models of clinical or other domain specific concepts, archetypes are } \\
\text { clinically meaningful entities. An EHR entry which has been archetyped will } \\
\text { have the same meaning no matter where or in which EHR it appears. Thus, } \\
\text { archetypes can be shared by multiple health systems and authorities, enabling } \\
\text { information to be shared between different systems and types of healthcare } \\
\text { professionals. Clinical knowledge can be shared and clinical information can } \\
\text { be safely interpreted by exchanging archetypes. }\end{array}$ \\
\hline $\begin{array}{l}\text { Semantic inter- } \\
\text { operability }\end{array}$ & $\begin{array}{l}\text { Domain Knowledge } \\
\text { Governance }\end{array}$ & $\begin{array}{l}\text { The use of archetypes and the reference model alone do not guarantee that } \\
\text { different EHR systems and vendors will construct equivalent EHR extracts, and } \\
\text { use the record hierarchy and terminology in consistent ways. Thus, this alone } \\
\text { does not ensure semantically interoperable systems. For semantically interoper- } \\
\text { able systems, archetype development must be coordinated through systematic } \\
\text { "Domain Knowledge Governance" to, for example, avoid incompatible, over- } \\
\text { lapping archetypes for essentially the same concept. }\end{array}$ \\
\hline
\end{tabular}


the archetype could be constrained by the appropriate subset of a terminology that deals with diagnoses.

Terms within an archetype can be bound to or constrained by any number of terminologies or, if highly specific to the archetyped concept, be specified within the archetype itself. Archetypes in contrast to terminologies specify groups of data that as a whole are whole, discreet, highly related and clinically meaningful concepts like a blood pressure measurement consisting of the diastolic and systolic blood pressure, but also of any other information relevant for the interpretation of the readings, e.g. the position of the patient during the measurement.

\subsubsection{The Relationship of openEHR and Archetypes to Standards}

Standards are one important part of the means to achieve our two aims (namely semantic interoperability and clinician empowerment). Based on the systematic comparison of openEHR specifications/reference implementations and existing archetypes with standards documents and drafts (see also [35]), this section aims to provide a summary on the relationship between openEHR and current standards and standards development activities.

Standards development work is continuing internationally to enable effective electronic communication as required for widespread EHRs adoption. In particular the European pre-standard, ENV 13606 Electronic Health Record Communication [36], origi-

nally a four-part standard is now being enhanced by the CEN EHRcom TaskForce to become a five-part full normative European standard (EN 13606 [37]). This is arguably the single most important standard requiring international consensus as it defines the fundamental EHR infrastructure requirements and will foster semantic interoperability in health. This standard is also in development for the International Organization for Standardization (ISO) [38] and it is intended that the five parts of EN13606 will be adopted as Australian Standards when they are completed in Europe. While
openEHR specifications are not being created as a formal consensus standard, over the past five years it has had a significant influence on the development of EHR standards by the three main international e-health standards development organisations, CEN (European Committee for Standardization), HL7 (Health Level 7), and ISO. In fact, CEN EN13606 is basically a subset of the full openEHR specification and one of the most important aspects of EN 13606 is the adoption of the openEHR two-level modeling approach (i.e. archetypes). CEN, openEHR and HL7 aim to harmonise this new standard with both openEHR (reference model and archetype approach) and with HL7 (mainly the Clinical Document Architecture).

Requirements for an Electronic Health Record Reference are standardised by ISO/ TS 18308. The purpose of this Technical Specification is to "assemble and collate a set of clinical and technical requirements for an electronic health record architecture [...]" [39]. It gives requirements for the architecture but does not specify the architecture (as openEHR does). The openEHR foundation under the leadership of Thomas Beale has prepared an openEHR/ISO 18308 Conformance Statement [40] detailing the compliance with each of the 123 requirements listed by the standard. One hundred and eight of the requirements are believed to be completely satisfied by the architecture either in a direct way, i.e. through a class or feature specifically designed for the requirement (96) or indirectly (12). The rest of the requirements are either partially fulfilled, scheduled for future openEHR versions or the compliance has yet to be determined.

Further, the openEHR approach is inline with ISO/DTR 20514 - Electronic Health Record Definition, Scope and Context [1]: In ISO/DTR 20514 the principle definition of the EHR is called the Integrated Care EHR (ICEHR). The ICEHR is based on a standardised or commonly agreed logical information model which supports semantic interoperability. The openEHR Reference Model and the CEN EN 13606 Reference Model are examples of models which fit this definition.

Lastly, the ISO 8601 standard [41] de- scribing formats for representing times, dates, and durations provides the syntax for all the date/time types defined in openEHR, including the partial ones. In the latest version openEHR also includes duration constraints based on ISO 8601.

In summary, openEHR and archetypes are therefore strongly related to existing standards and current standards developments. One concern in moving forward is the need for convergence of EHR and EHRrelated standards that seems to be required to realize true interoperability of health care applications due to the abundance of different EHR-related standards and specifications [42]. Fortunately, collaboration by the major players in this area is very good.

\subsection{Domain Knowledge Governance}

We define "domain knowledge governance as comprising all tasks related to establishing or influencing formal and informal organizational mechanisms and structures in order to systematically influence the building, dissemination, and maintaining of knowledge within and between domains" [43].

A fundamental aim of the archetype approach, as discussed above, is to empower domain experts to create and change the knowledge inherent in archetypes, thus controlling the way EHRs are built up using designed structures to express the required clinical data and assuring that all necessary constraints on the values of record components are observed. Most of the core set of archetypes has to be built by a relatively small group of clinicians. Individual groups of clinicians can then easily extend and adapt these archetypes to their needs. The openEHR Archetype Editor was built as a tool to support the domain experts' development of archetypes by providing them with an easy-to-use graphical user interface making the abstract openEHR archetype model tangible. As a consequence, health professionals can and need to take charge of managing their domain knowledge. However for the following reasons, archetype development will have to be coordinated:

- There will be significant concept overlaps between the various health care do- 
mains. For example, it may be that a nursing archetype for an oral assessment is equally applicable to knowledge domains other than nursing or that various nursing fields have different needs.

- Some archetypes need to be standardised as they are relevant for and need to be semantically interoperable between the various health areas and specialist fields and also between various organisations. It has to be defined what these archetypes are and who is responsible for them.

- Archetypes need to be easily accessible (e.g. on an internet server) and easily locatable, i.e. organised in a way that a certain archetype can be retrieved with high recall and precision from all existing archetypes.

- Archetypes have to be evidence-based whenever possible: Knowledge in archetypes needs to be based on evidence or best practice although it can include 'tacit' knowledge acquired by experts and agreed to by consensus.

- Archetypes need to be maintained and systematically updated when knowledge changes.

- More theoretically, Kumar and colleagues [44] and Pisanelli and Gangemi [45] argue that the adoption of an ontological framework facilitates the building of better and more interoperable information systems. It facilitates unambiguous communication of complex and detailed concepts. A domain ontology catalogues various aspects known to exist within the domain of interest from a specific point of view to suit a defined purpose. An ontological domain knowledge model shows how key concepts relate to one another and is usually based on a philosophical or theoretical foundation.

For these reasons, archetype development and maintenance has to be systematically organised - through the adoption of systematic domain knowledge governance. This has recently been supported by Australia's National E-Health Transaction Authority (NEHTA) stating that 'undisciplined creation and application of archetypes threatens the goal of semantic interoperability' [46]. Domain knowledge gov- ernance will ensure that archetypes will meet the information needs of the various areas, redundancy is minimised and semantic interoperability vastly increased.

Figure 1 shows the regions of standardisation for archetypes and the scope of archetype standardisation. Domains for domain knowledge governance can be widereaching sectors such as health care, or discipline areas such as medicine or nursing, or fields and sub-fields like midwifery or paediatric oncology. Archetypes can be standardised for different regions from single provider organisations to world-wide standardisation. The scope of archetype standardisation can reach from sub-field or field (e.g. paediatric oncology), and discipline area (e.g. medicine) to the sector (health), and can also be relevant beyond health for other sectors. Not always is it desirable to

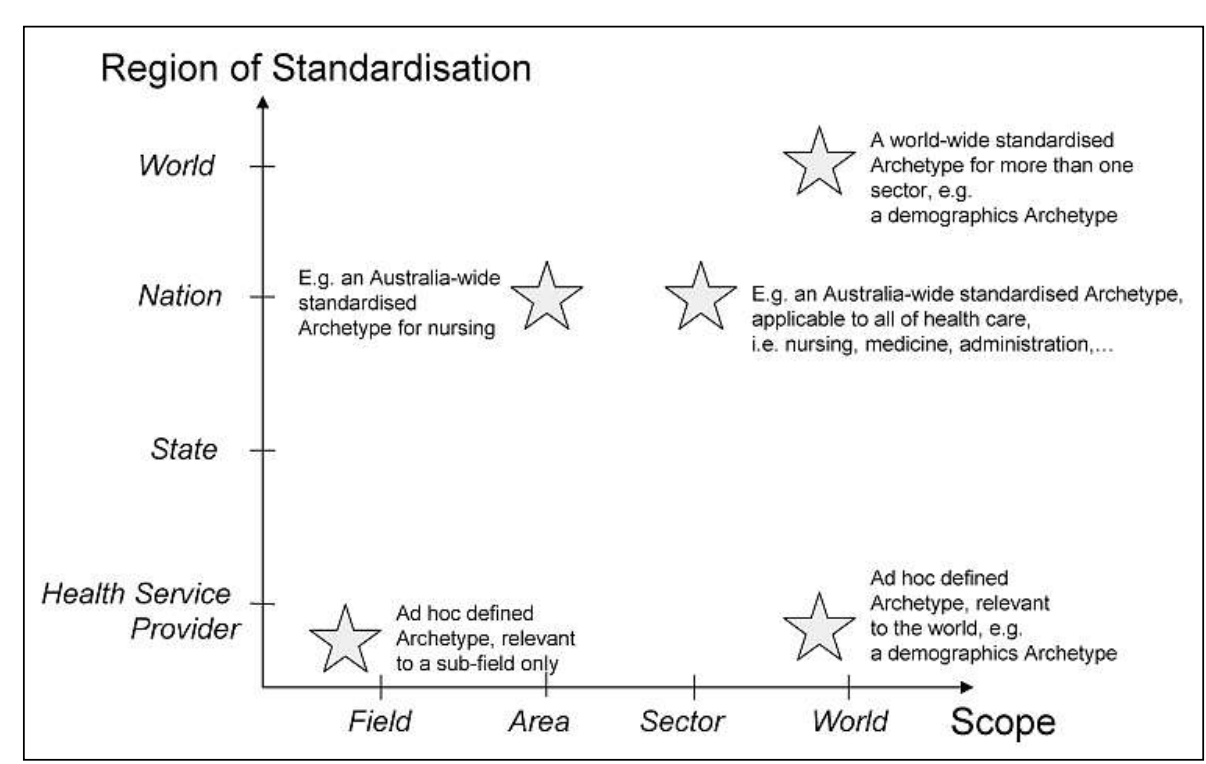

Fig. 1 The realm of Domain Knowledge Governance: region of standardisation and scope of archetypes

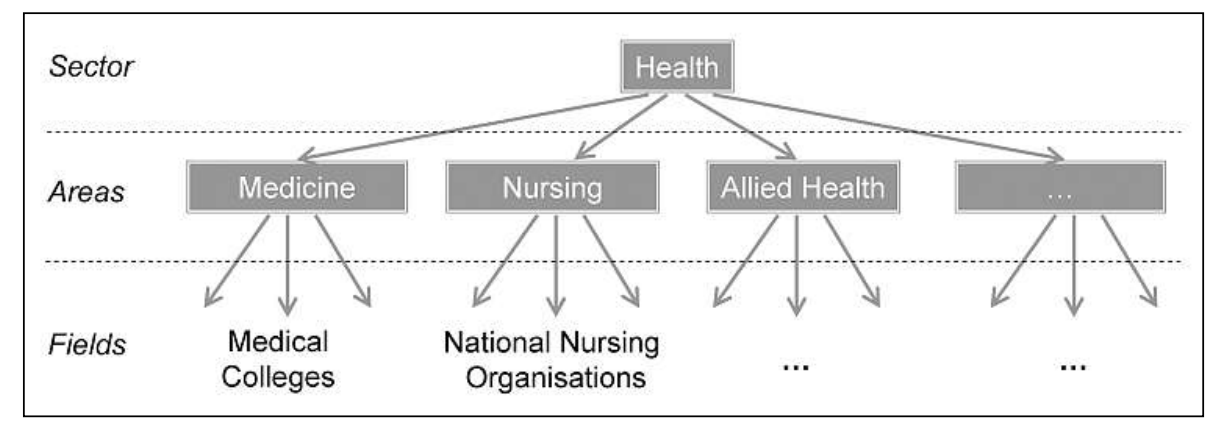

Fig. 2 Overview of a top-down-approach to Domain Knowledge Governance in health

strive for worldwide standardisation and worldwide scope. Rather, many archetypes will only be relevant for one field or one area - but this has to be ascertained by the various domain levels. In many cases it will also make sense to specialise or localise an existing health-care-wide and nation-wide standardised archetype to suit a specific field or a specific health service provider.

Figure 2 presents an overview of a topdown-approach to domain knowledge governance in health. Essentially an approach is required that ensures that archetypes will be compatible, comprehensive, and not overlapping inconsistently. For this, we need to identify archetypes that are to be used by different discipline areas in health, so that these conflicts can be resolved or - better prevented in the first place. Then on the next level, medicine has to ensure that archetype 


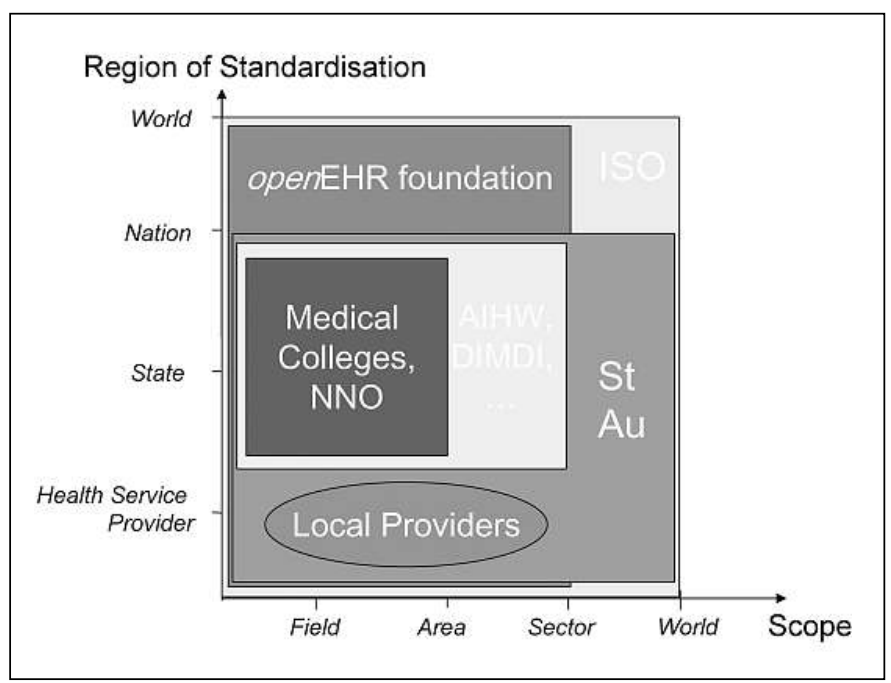

Fig. 3

Domain Knowledge Governance - who should be responsible for which part of the realm? The figure provides an overview of one possible - although simplified - solution. The figure is presented from an Australian point of view. St Au stands for Standards Australia, AlHW is the Australian Institute for Health and Welfare, NNO the National Nursing Organisations group, and ISO the International Standardisation Organisation. work in different specialist fields (e.g. by medical colleges) is compatible, while nursing and possibly other areas have to do the same for their area. This will constantly be interwoven with the effort to broaden the region of standardisation.

Figure 3 shows one scenario of organisations that could be responsible for the regions and scope of archetype standardisation. Organisations such as the Australian Institute of Health and Welfare, DIMDI the German Institute for Medical Documentation and Information, or the Canadian Institute for Health Information may well be in the best position for national Domain Knowledge Governance, but it would require a change to their existing organisational mission and operations. Internationally, the openEHR Clinical Review Board could fulfil this position or organisations such as the Cochrane collaboration, an international not-for-profit organisation, providing up-to-date information about the effects of health care. It is highly desirable to use an existing organisation of this kind to manage archetypes and to make these available. Archetypes that reflect the knowledge of specific clinical knowledge domains can only be defined and maintained by the profession that specialises in that area of practice. In medicine, the professional colleges are in charge of their specific knowledge domains, while in nursing, the National Nursing Organisations (NNO) group, now consisting of more than 50 nursing organisations could coordinate the development of nursing archetypes for Australia [47]. An interdisciplinary team needs to be established on sector-level that define what archetypes need to be developed and by which intra- or interdisciplinary teams on area or field level. For this domain experts must be identified and trained. We need to identify individual ontological knowledge domains, establish who governs which knowledge domain and assess priorities for archetype development. Further, a process for engaging knowledge domain experts, for the development or specialisation of archetypes on area and field level has to be defined. Healthwide archetypes can be specialised for various areas, and likewise a nursing area archetype (e.g. an oral assessment) can be specialised for a field (e.g. an oncology oral assessment) without endangering semantic interoperability. We need a formalised process to ensure that archetype design principles are adhered to. This is currently formalised by European and international standardisation activities (e.g. [48]).

\subsection{Supporting Domain Know- ledge Governance with Information Technology}

A framework for managing archetypes, identifying which need to be standardised, which are domain-specific and establish and train multidisciplinary teams for archetype development and coordination will inform and support Domain Knowledge Governance. To support this we employed the Web Ontology Language (OWL [25]) and the Protégé OWL Plug-In [26] to develop and maintain an Archetype Ontology which provides the necessary metainformation on archetypes for Domain Knowledge Governance. The Archetype Ontology captures the meta-information about archetypes needed to support Domain Knowledge Governance. This includes information such as the EHR class of the archetype (for example: Observation, Evaluation, Instruction), the purpose of the archetype (for example Chronic Disease Management), or the age group of the patient to which the archetype is applicable. Some of this information, such as the EHR class, is expressed within the archetype itself; other information, such as the age group, can only be formally expressed in the Protégé knowledge base. Also, the domain for which the archetype is relevant is included (for example medicine and nursing and their sub-categories).

Based on this ontology we developed a web-based Archetype Finder ${ }^{\mathrm{b}}$. The Archetype Finder supports the user in easily locating and accessing archetypes. A screenshot of the Archetype Finder is given in Figure 4. This user interface is generically built based on the Archetype Ontology, i.e. changes in structure and content of the Archetype Ontology are immediately reflected in the Archetype Finder without modifications to the softwae. After specifying the requested properties of archetypes, the Archetype Finder searches the ontology and presents an overview of all matching archetypes. Figure 5 presents an overview of what the Archetype Finder presents when searching the Blood Pressure Archetype from our running example.

Usage experience in Australia shows that the Archetype Finder is one of the fastest ways to locate and access archetypes. As query, search and presentation are all ontology-based, the Archetype Finder is a flexible tool that can relatively easy be adapted to reflect evolving needs. The ontologybased nature of the Archetype Finder also

\footnotetext{
b The Archetype Finder is available online at http://www.archetypes.com.au
} 
enables the automatic search for archetypes by information systems, for example a system could dynamically search for all archetypes for a female, 50-year-old female that are relevant for the purpose of chronic disease management in Australia. Such a query would - among others - return the blood pressure archetype to the system. Current limitations of the Archetype Finder include that no concept of vicinity (other than mono-hierarchies) is included, for example the system cannot decide to what extent an archetype that is defined relevant for Paediatric Oncology is also relevant for Oncology and/or Paediatrics in general or vice versa.

The Archetype Finder is currently being expanded to a comprehensive Archetype Repository and will also include further mechanisms to support Domain Knowledge Governance with a clear process for authoring, updating, managing, disseminating knowledge in archetypes as well as archetype version control.

\subsection{Real World Examples}

Domain experts have worked with this approach and continue to do so. For example, a series of workshops on archetype development for clinicians were conducted by two of the authors (EH, SG) and among many other activities an archetype road show was conducted ( $\mathrm{SH}$ ). Archetypes have been developed for the General Practice Computing Group Australia (GPCG). The set of archetypes developed for the GPCG is available in the Archetype Finder (http://www.archetypes.com.au), more openEHR archetypes are available from the openEHR website. Australia's National EHealth Transaction Authority (NEHTA) a joint initiative established by the Australian State and Territory governments works with archetypes for the definition of Clinical Data Standards [49]. Nursing domain experts work with this approach, and the authors currently work together with researchers and clinicians in several countries including Australia, Turkey, Germany and Iran to define and refine archetypes for various areas including Aged Care and Pediatric Oncology.

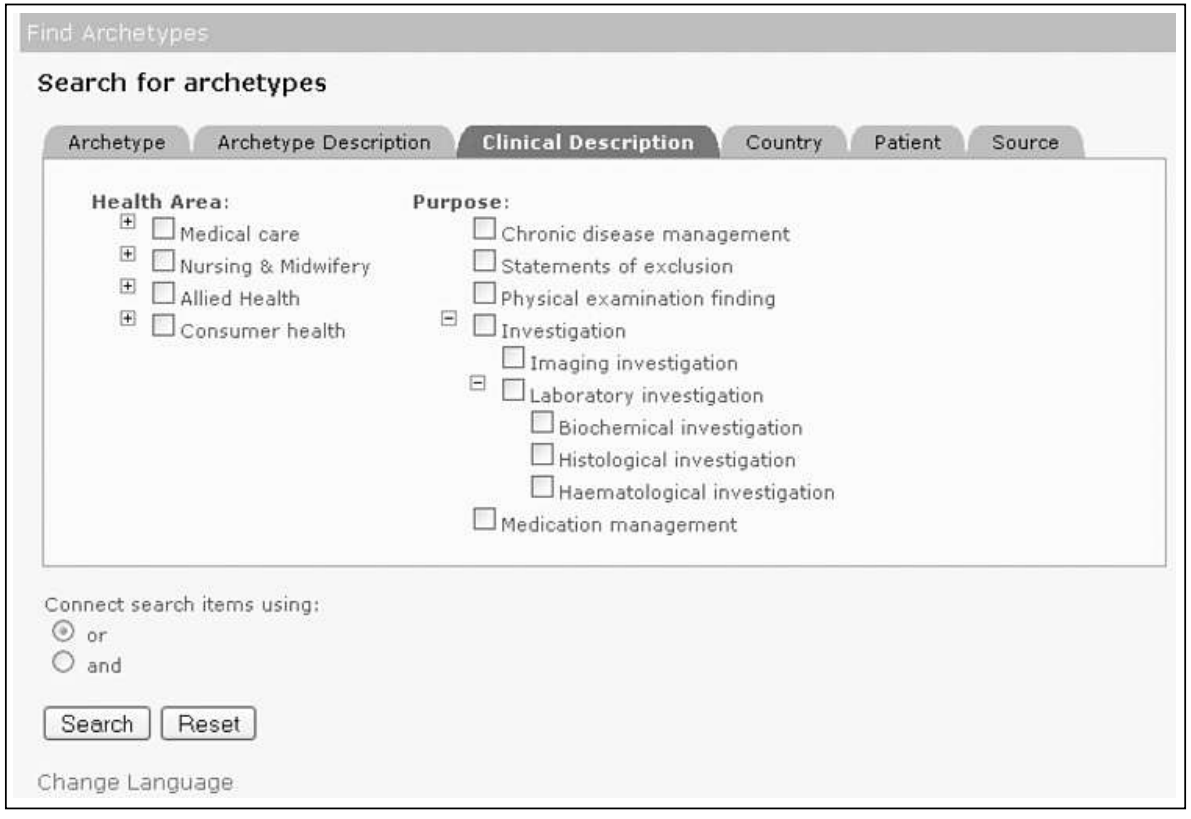

Fig. 4 Screenshot from the Archetype Finder. The Archetype Finder searches for available archetypes based on the Archetype OWL Ontology.

\begin{tabular}{|c|c|}
\hline \multicolumn{2}{|l|}{ Find Archetypes } \\
\hline \multicolumn{2}{|c|}{1 Archetype(s) found } \\
\hline \multicolumn{2}{|c|}{ You can now narrow or broaden your search result or start a new search. } \\
\hline Search & Query \\
\hline Search 1 & Archetype ID = 'blood_oressure' \\
\hline \multicolumn{2}{|c|}{ Blood pressure measurement } \\
\hline Archetype ID & openEHR-EHR-OBSERVATION.blood_pressure.v1 \\
\hline Description & $\begin{array}{l}\text { the measurement of systemic arterial blood pressure which is } \\
\text { deemed to represent the actual systemic blood pressure }\end{array}$ \\
\hline EHR class & Observation \\
\hline Health Area & Patient recording \\
\hline Purpose & $\begin{array}{l}\text { Physical examination finding } \\
\text { Chronic disease management }\end{array}$ \\
\hline Country & Australia \\
\hline Data source & Observation \\
\hline \multicolumn{2}{|l|}{ Links } \\
\hline HTML & openEHR-EHR-OBSERVATION.blood_pressure.v1.html \\
\hline ADL & openEHR-EHR-OBSERVATION.blood_pressure.v1.adl \\
\hline
\end{tabular}

Fig. 5 Screenshot of the Archetype Finder displaying the result set for the archetype search: "Archetype ID = "blood pressure'. It presents the archetype ID, the concept description and the meta-information on the archetypes. It further hyperlinks to a non-technical HTML presentation of the archetype and to the Archetype as specified by the Archetype Definition Language (ADL).

The Brisbane Southside Trial of Australia's national HealthConnect initiative to create a nationwide EHR network (http://healthconnect.gov.au) employed openEHR and archetypes [24], as well as the New South Wales Cancer Registry Information Technology Online (ITOL) Program. While Australia's National E-Health Transaction Authority (NEHTA) concluded that " $[. .$.$] all potential architectures (includ-$ 
ing EN13606, HL7v3 and openEHR) are unproven in large-scale Shared EHR implementations" [46], the HealthConnect Trial was one important step into that direction for openEHR. NEHTA recommends in the same report to "[a]dopt the European EN13606 standard on EHR Communication (parts 1 to 3 ) as the basis of an Australian Shared EHR Architecture Standard for specifying Australian Shared EHR Content". As noted in Section 4.2.2, EN 13606 is based as a subset of the full openEHR specification and one of the most important aspects of EN 13606 is the adoption of the openEHR two-level modeling approach (i.e. archetypes). Further examples include the experiences in two field trials supported by the Australian General Practice Computing Group (GPCG) [50].

Apart from many openEHR research activities around the globe, the foundation's specifications are also being implemented in commercial deployments ${ }^{\mathrm{c}}$ for example in the Netherlands, Sweden, the USA, Belgium, and Australia [51].

\section{Discussion}

It is clear that internationally there is a strong desire to develop and implement semantically interoperable health information systems and EHRs. This has for example been formalised by a World Health Organisation (WHO) resolution on eHealth adopted at the 58th World Health Assembly in May 2005 [52].

The traditional method of clinical system development is to collect functional requirements, usually from a small number of 'typical' clinicians. Then the software is designed based on a single large model which incorporates both the generic business rules for the system and the domain-specific (clinical) requirements. As sophisticated as this requirements engineering may be (e.g. [53]), the 'impedance mismatch' between IT specialists and clinicians, the complexity of clinical systems and the variability of work patterns between different clinicians, often means that the resulting clinical sys-

\footnotetext{
See http://openehr.org/projects/t_projects.htm for up-to-date information on these deployments.
}

tem is an inflexible 'one size fits all' compromise which in reality suits no-one [3]. We therefore believe that we have to go one step further than performing precise activity and requirement analyses and make the clinician ultimately control - not only influence - the clinical content of health information systems like EHR systems. Otherwise, the concern is that no clinical value is added. Current clinical information systems directly incorporate clinical knowledge. The use of archetypes means that EHR software can now be considerably smaller than traditionally and requires little maintenance since all knowledge will reside in softwareindependent archetypes allowing changes to be made without altering software or databases, based on a small and stable information model. This also enables the EHR to increasingly satisfy user needs, rather than degrading into obsolescence, as invariably happens with today's health systems. Also, the greater flexibility during standardisation processes offered by openEHR archetyping is of great value during knowledge harmonization and governance.

Although we argue in this paper that Domain Knowledge Governance should be a formal process, Domain Knowledge Governance can also be more informal. However formal or informal, it is clear that Domain Knowledge Governance is needed independent from the actual EHR approach chosen: without clinical knowledge being adequately defined, maintained and standardised, in our conviction, (patientcentred, longitudinal, comprehensive, and prospective) EHRs are not achievable. Other approaches like HL7 v3 or the Clinical Document Architecture (HL7 CDA, [54, 55]) will need to do essentially the same if semantic interoperability is to be achieved between more than two systems at a time. For this, the standardisation of the HL7 Reference Information Model (RIM) for example is not sufficient; rather the standardisation of R-MIMs and use of templates is required and ideally related to archetypes. By clearly separating technical and clinical concerns, the openEHR and archetypes methodology offers a good way to define and maintain the knowledge inherent in EHRs and support Domain Knowledge
Governance. Recent experiences with nurses by two of the authors (SG, EH) at two nursing archetype workshops in Melbourne and Brisbane $[56,57]$ also show how valuable archetypes are to get nurses involved in defining their nursing knowledge. The developed Archetype Ontology as well as the Archetype Finder are in harmony with standardisation efforts by ISO and CEN for an 'Archetype Management Framework' as proposed by Dipak Kalra and Thomas Beale [48].

While HL7 v.3 primarily serves as a framework to define messages between applications and HL7 CDA is a generic model for the communication of clinical documents, and in this is similar to openEHR Compositions, openEHR's focus is the EHR as a whole. Oemig and Blobel argue in [58] that HL7 is moving towards an architecture standard. The openEHR approach however differs from message-based approaches in two very important ways:

- The data model is fixed and data easily shared regardless of which archetype is used.

- Communications can be created ad hoc to meet the immediate needs of the clinicians as long as they are communicating archetyped data.

In HL 7 v.3, two means are used to constrain a generic Reference Information Model (RIM): a constrained version of RIM classes creating a specific information model Refined Message Information Model (RMIM) - and secondly the proposed HL7 Templates [59]. The new CDA release 2 requires HL 7 Templates (once developed) to constrain the document sections based on the type of document (section-level templates) and to constrain the entries within document sections (entry-level templates). Similarly, the German SCIPHOX project (http://www.sciphox.de [60]) uses 'Small Semantic Units' to specify CDA documents and their structures. NEHTA's review of Shared Electronic Health Record Standards offers a more in-depth discussion of similarities and differences between these approaches [46]. Two issues however are important in order not to endanger semantic interoperability:

- First, it is essential to strive for harmonization between all the approaches to the 
Domain Knowledge Governance for openEHR Archetypes

greatest extent possible (for example by using the same data types adhering to existing standards). Harmonisation between HL7 v.3, HL7 CDA, openEHR and standards like CEN ENV 13606 [36] is ongoing: A combination of good working relationships between representatives from CEN, openEHR and HL7 has led to efforts to harmonise this standard with HL7 (RIM, CDA and Templates) and with openEHR (reference model and archetype approach). The three groups are developing crossmappings to enable the exchange of EHR data between implementations of each approach $[35,61]$.

- Second, it is necessary to define precisely when to use which approach as it is for example done in Australia by NEHTA's review of Shared Electronic Health Record Standards [46].

Archetype development requires standards developers to work more closely with domain knowledge experts and means a paradigm shift regarding the methods adopted. Archetype development and maintenance require the adoption of principles not unlike those used for the development of a classification system or terminology. This requires the adoption of a set of processes that enable the creation, development, organisation, sharing, dissemination, use and continuous maintenance of archetypes. Many archetypes need to be standardised, managed and maintained by the relevant domain knowledge experts.

The openEHR two-level modelling and archetype methodology cannot overcome all of the barriers to more efficient use of computerised clinical systems. Easier and faster data capture at the point of care remains a major challenge, which we will only be able to overcome by a combination of more efficient technologies such as voice or handwriting recognition (e.g. [62]). The openEHR approach can however make a significant contribution to the flexibility and usability of clinical systems and to the improvement of data quality and semantic interoperability and thus also serve as the key enabler for intelligent decision support, care planning and other value-adding applications. Data security and data protection issues as e.g. considered by van der Haak et al.
[63] taking into account who is entitled to view, update and logically or physically delete the information provided and the fact that sharing of patient-related information between different providers may cause legal, ethical and cultural problems in various countries will always have to be considered when implementing EHRs. Especially, patient data used for research activities often is a complex (legal) issue [64]. On a different note, advanced data formats used (e.g. pictures) need to be suitable for conclusive and secure long-term archiving (cp. [65]). In the future, genomic data is likely to be integrated into EHRs and we may have to deal with data types that are possibly not yet sufficiently standardised for EHR integration, we also need to link genotype and phenotype data appropriately; again confidentiality and privacy concerns have to be addressed for this very sensitive data [66].

Finally, one key aspect of EHRs is that they have to be available throughout the life of the patient - sustainability therefore is a major issue. Shabo's global socioeconomic-medico-legal model for the sustainability of longitudinal electronic health records using independent health record banks [67] is independent from any particular technical basis for EHRs. Shabo's model requires true semantic interoperability and we believe that openEHR and archetypes would provide an excellent basis for such a sustainability model.

\section{Conclusion}

To enable (life-long) EHRs, semantic interoperability is of major importance. The openEHR archetypes approach enables syntactic interoperability and semantic interpretability. The definition of archetypes is an important step for the realisation of EHRs and achieving semantic interoperability. To enable true and comprehensive semantic interoperability, the development and maintenance of archetypes needs to be coordinated and governed internationally and across the various health professions. However, archetype development and maintenance needs to be supported and governed by national and international processes
(Domain Knowledge Governance) and supported adequately by Information Technology, otherwise 'rank growth' of archetypes will jeopardize semantic interoperability. Collectively, we believe that this will create the knowledge environment required to adopt semantically interoperable EHRs.

\section{Acknowledgements \\ The development of the Archetype Finder was sup- ported by the Australian General Practice Computing Group through funding from the Australian Depart- ment of Health and Ageing. Further, the development was supported by an internal research grant of Central Queensland University's former Faculty of Infor- matics and Communication.}

\section{References}

1. International Organisation for Standardisation (ISO). 20514 Draft Technical Report: EHR Definition, Scope and Context.

2. Ash JS, Bates DW. Factors and forces affecting EHR system adoption: report of a 2004 ACMI discussion. J Am Med Inform Assoc 2005; 12 (1): 8-12.

3. Schloeffel P. openEHR archetypes: Putting the clinician back in the driver's seat. HIC 2003 (Health Informatics Conference Australia), Sydney, 2003.

4. UK Royal College of Nursing. Nurses and NHS IT developments. 2005. http://www.rcn.org.uk/ downloads/research/nurses-it-devs-survey.doc (Last accessed: 08.08.2006).

5. Kuhn KA, Giuse DA. From hospital information systems to health information systems. Problems, challenges, perspectives. Methods Inf Med 2001; 40 (4): 275-87.

6. van Bemmel JH,Musen MA. Handbook of Medical Informatics. Heidelberg: Springer; 1997.

7. Brandner R, van der Haak M, Hartmann M, Haux R, Schmücker P. Electronic Signature for Medical Documents - Integration and Evaluation of a Public Key Infrastructure in Hospitals. Methods Inf Med 2002; 41 (4): 321-30.

8. Frankel A, Gandhi TK, Bates DW. Improving patient safety across a large integrated health care delivery system. Int J Qual Health Care 2003; 15 (Suppl 1): i31-i40.

9. Classen DC, Kilbridge PM. The roles and responsibility of physicians to improve patient safety within health care delivery systems. Acad Med 2002; 77 (10): 963-72.

10. Fitzpatrick NK, Shah S, Walker N, Nourmand S, Tyrer PJ, Barnes TR, Higgitt A, Hemingway H. The determinants and effect of shared care on patient outcomes and psychiatric admissions - an inner city primary care cohort study. Soc Psychiatry Psychiatr Epidemiol 2004; 39 (2): 154-63.

11. Smith S, Bury G, O'Leary M, Shannon W, Tynan A, Staines A, Thompson C. The North Dublin ran- 
domized controlled trial of structured diabetes shared care. Fam Pract 2004; 21 (1): 39-45.

12. Harris MF, Priddin D, Ruscoe W, Infante FA, O'Toole BI. Quality of care provided by general practitioners using or not using Division-based diabetes registers. Med J Aust 2002; 177 (5): 250-2.

13. Ciardullo AV, Daghio MM, Brunetti M, Bevini M, Daya G, Feltri G, Novi D, Goldoni CA, Guerzoni A, Messori A, Magrini N. Audit of a shared-care program for persons with diabetes: baseline and 3 annual follow-ups. Acta Diabetol 2004; 41 (1): 9-13.

14. Kuperman GJ, Spurr C, Flammini S, Bates D, Glaser J. A clinical information systems strategy for a large integrated delivery network. Proc Amia Symp 2000: 438-42.

15. Wan TT, Lin BY, Ma A. Integration mechanisms and hospital efficiency in integrated health care delivery systems. J Med Syst 2002; 26 (2): 127-43.

16. Wang SJ, Middleton B, Prosser LA, Bardon CG, Spurr CD, Carchidi PJ, Kittler AF, Goldszer RC, Fairchild DG, Sussman AJ, Kuperman GJ, Bates DW. A cost-benefit analysis of electronic medical records in primary care. Am J Med 2003; 114 (5): 397-403.

17. Safran C. The collaborative edge: patient empowerment for vulnerable populations. Int J Med Inf 2003; 69 (2-3): 185-90.

18. Garde S, Wolff AC, Kutscha U, Wetter T, Knaup P. CSI-ISC: Concepts for Smooth Integration of Health Care Information System Components into Established Processes of Patient Care. Methods Inf Med 2006; 45 (1): 10-8.

19. Ammenwerth E, Shaw NT. Bad health informatics can kill - is evaluation the answer? Methods Inf Med 2005; 44 (1): 1-3.

20. Shabo A, Vortman P,Robson B. Who's Afraid of Lifetime Electronic Medical Records. In: Proceedings of 1st Annual Conference on Mobile and Wireless Healthcare Applications, November 11-14, 2001. Café Royal, London.

21. Cimino JJ. Terminology tools: state of the art and practical lessons. Methods Inf Med 2001; 40 (4): 298-306.

22. Mludek V, Knaup P, Garde S, Merzweiler A, Weber R, Wetter T. Formale Definition von Integritätsbedingungen für den Basisdatensatz der Pädiatrischen Onkologie (Formal Definition of Integrity Constraints for a Basic Data Set for Pediatric Oncology). Informatik, Biometrie und Epidemiologie in Medizin und Biologie 2002; 33 (2-3): 338

23. Xu Y, Sauquet D, Degoulet P,Jaulent MC. Component-based mediation services for the integration of medical applications. Artif Intell Med 2003; 27 (3): 283-304.

24. Goodchild A, Gibson K, Anderson L, Bird L. The Brisbane Southside HealthConnect Trial: Preliminary Results. Health Informatics Conference (HIC), Brisbane, 2004.

25. W3C. OWL Web Ontology Language Overview - W3C Recommendation 10 February 2004. 2004. http://www.w3.org/TR/owl-features/ (Last accessed: 08.08.2006).
26. Knublauch H, Fergerson RW, Noy NF, Musen MA. The Protégé OWL Plugin: An Open Development Environment for Semantic Web Applications. Third International Semantic Web Conference - ISWC 2004.

27. Rector AL. Terminology and concept representation languages: where are we? Artif Intell Med 1999; 15 (1): 1-4.

28. Beale T. Archetypes: Constraint-based Domain Models for Future-proof Information Systems. OOPSLA 2002 workshop on behavioural semantics, 2002.

29. Veltman KH. Syntactic and Semantic Interoperability: New Approaches to Knowledge and the Semantic Web. The New Review of Information Networking 2001; 7: 159-84.

30. Institute of Electrical and Electronics Engineers (IEEE). IEEE Standard Computer Dictionary: A Compilation of IEEE Standard Computer Glossaries. New York, NY, 1990.

31. Sheth AP. Changing Focus on Interoperability in Information Systems: From System, Syntax, Structure to Semantics. In: Goodchild MF, Egenhofer MJ, Fegeas R, Kottman CA (eds). Interoperating Geographic Information Systems. Norwell, MA: Kluwer; 1999.

32. Elkin PL, Brown SH, Lincoln MJ, Hogarth M, Rector A. A formal representation for messages containing compositional expressions. Int J Med Inform 2003; 71: 89-102.

33. Health Level 7. HL7 EHR System Functional Model: A Major Development Towards Consensus on Electronic Health Record System Functionality - A White Paper. 2004. http://www. hl7.org/ehr/downloads/dstu/EHR-SWhitePaper. zip (last accessed: 08.08.2006).

34. Min Z, Baofen D, Weeber M,van Ginneken AM. Mapping OpenSDE domain models to SNOMED CT. Applied to the domain of cardiovascular disease. Methods Inf Med 2006; 45 (1): 4-9.

35. Kalra D. Electronic Health Record Standards. In: Haux R, Kulikowski C, editors. IMIA Yearbook of Medical Informatics 2006. Methods Inf Med 2006; 45 Suppl 1: S136-44.

36. CEN. ENV 13606 Health informatics -Electronic health record communication. 1999

37. CEN. ENV 13606-1 Health informatics - Electronic health record communication - Part 1: Reference model. 1999.

38. ISO. Committee Draft (CD) 13606-1 Health informatics - Electronic health record communication - Part 1: Reference model.

39. International Organisation for Standardisation (ISO). ISO/TS 18308: Requirements for an Electronic Health Record Reference Architecture. 2004

40. Beale T. openEHR/ISO 18308 Conformance Statement. The openEHR Foundation. 2006. http://svn.openehr.org/specification/TRUNK/ publishing/requirements/iso18308_conformance. pdf (last accessed: 08.08.2006)

41. International Organisation for Standardisation (ISO). ISO 8601, Data elements and interchange formats - Information interchange - Representation of dates and times. 1988.
42. Jaspers MWM, Knaup P, Schmidt D. The Computerized Patient Record: Where Do We Stand? In: Haux R, Kulikowski C, editors. IMIA Yearbook of Medical Informatics 2006. Methods Inf Med 2006; 45 (Suppl 1): S29-39.

43. Garde S, Heard S, Hovenga E. Archetypes in Electronic Health Records: Making the case and showing the path for domain knowledge governance. In: Grain H, Wise M (eds). HIC 2005: 13th Australian Health Informatics Conference, Melbourne, 31.07.2005-02.08.2005, Brunswick East, Vic: Health Informatics Society of Australia; 2005.

44. Kumar A, Smith B, Pisanelli DM, Gangemi A, Stefanelli M. An Ontological Framework for the Implementation of Clinical Guidelines in Health Care Organisations. In: Pisanelli DM (ed). Ontologies in Medicine. Amsterdam: IOS Press; 2004.

45. Pisanelli DM, Gangemi A. If Ontology is the Solution, What is the Problem? In: Pisanelli DM (ed). Ontologies in Medicine. Amsterdam: IOS Press; 2004.

46. National E-Health Transition Authority (NEHTA). Review of Shared Electronic Health Record Standards - Version 1.0. 2006. http:// www.nehta.gov.au/component/option, com_ docman/task,doc_download/gid,68/Itemid, 139/ (last accessed: 08.08.2006).

47. Hovenga E, Garde S, Heard S. Nursing Constraint Models for Electronic Health Records: a vision for domain knowledge governance. Int J Med Inf 2005; 74: 886-98.

48. CEN. Archetype Knowledge Framework - accepted as new work item. 2005.

49. National E-Health Transition Authority (NEHTA). Clinical Data Standards: Adverse Reaction and Alert Archetype Representations. Final Report. 2005. http://www.nehta. gov.au/component/option,com_docman/task, doc_download/gid,35/Itemid,139/ (last accessed: 08.08.2006).

50. Bird L, Goodchild A, Tun Z. Experiences with a Two-Level Modelling Approach to Electronic Health Records. Journal of Research and Practice in Information Technology 2003; 35 (2).

51. Al-Ubaydli M. Open source medical records systems around the world. UK Health Informatics Today 2006; 49 (3-4)

52. WHO. WHA58.28 - Resolution on eHealth. 58th World Health Assembly. 2005. http://www.who. int/gb/ebwha/pdf_files/WHA58/WHA58_28-en. pdf (last accessed: 08.08.2006).

53. Beuscart-Zéphir MC, Anceaux F, Crinquette V, Renard JM. Integrating user's activity modeling in the design and assessment of hospital electronic patient records: the example of anesthesia. Int J Med Inf 2001; 64 (2-3): 157-71.

54. Dolin RH, Alschuler L, Beebe C, Biron PV, Boyer SL, Essin D, Kimber E, Lincoln T, Mattison JE. The HL7 Clinical Document Architecture. J Am Med Inform Assoc 2001; 8 (6): 552-69.

55. Blobel B. Advanced and secure architectural EHR approaches. Int J Med Inform 2005; 75 (3-4): 185-90.

56. Garde S. Developing and Managing Archetypes. NIA - HISA Nursing Informatics SIG Workshop: 
Capturing Health Knowledge: developing archetypes, July 1, 2005 Brisbane, 2005.

57. Garde S, Hovenga E, Conrick M. Integrating nursing concepts into Electronic Health Records. Nursing Informatics Australia Conference: "Nurses make a difference: using information to make nurses' contribution visible". Melbourne, December 4, 2004

58. Oemig F, Blobel B. Does HL7 Go towards an Architecture Standard? Stud Health Technol Inform 2005; 116: 761-6.

59. Heard S, Beale T, Freriks G, Mori AR, Pishev O. Templates and Archetypes: how do we know what we are talking about? 2003. http://www.openehr. org/downloads/archetypes/templates_and_ archetypes.pdf (last accessed: 08.08.2006).

60. Heitmann KU, Schweiger R, Dudeck J. Discharge and referral data exchange using global standard the SCIPHOX project in Germany. Int J Med Inform 2003; 70 (2-3): 195-203.
61. CEN/TC 251. EHRCOM prEN 13606-1. Health informatics - Electronic health record communication - Part 1: Reference model. 2005.

62. Despont-Gros C, Landau R, Rutschmann O, Simon J, Lovis C. The digital pen and paper. Evaluation and acceptance of a new data acquisition device in clinical settings. Methods Inf Med 2005; 44 (3): 359-68.

63. van der Haak M, Wolff AC, Brandner R, Drings $P$, Wannenmacher M, Wetter T. Data security and protection in cross-institutional electronic patient records. Int J Med Inf 2003; 70 (2-3): 117-30.

64. Mulligan E, Rogers W, Braunack-Mayer A. Research within the Privacy Regulations: Problems and Solutions for Database Custodians. Electronic Journal of Health Informatics 2006; 1 (1): e7. http://ejhi.net/ojs/index.php/ejhi/article/ view/e7 (last accessed: 08.08.2006).

65. Hollerbach A, Brandner R, Bess A, Schmucker P, Bergh B. Electronically signed documents in health care - analysis and assessment of data formats and transformation. Methods Inf Med 2005; 44 (4): 520-7.

66. Sax U, Schmidt S. Integration of genomic data in Electronic Health Records - opportunities and dilemmas. Methods Inf Med 2005; 44 (4): 546-50.

67. Shabo A. A global socio-economic-medico-legal model for the sustainability of longitudinal electronic health records. Part 1. Methods Inf Med 2006; 45 (3): 240-5.

\section{Correspondence to:}

Dr. Sebastian Garde

Faculty of Business and Informatics

Central Queensland University

Austin Centre for Applied Clinical Informatics, Austin Health Heidelberg VIC 3084

Australia

E-mail:s.garde@cqu.edu.au 